\title{
Circulating levels of Galectin 9 are a potential biomarker predictive of overall survival in patients with advanced Non-Small-Cell Lung Cancer
}

\section{Guilherme Jorge Costa}

Hospital de Câncer de Pernambuco

Guilherme Vieira Mendonça Filho

Instituto de Medicina Integral Professor Fernando Figueira (IMIP)

Leuridan Cavalcante Torres ( $\square$ leuridan.torres@gmail.com )

Instituto de Medicina Integral Professor Fernando Figueira (IMIP)

Dulce Elena Casarini

Federal University of São Paulo

\section{Research Article}

Keywords: TIM-3 protein, galectin-9, Non-Small-Cell Lung Carcinoma, T cells, smoking

Posted Date: February 10th, 2022

DOI: https://doi.org/10.21203/rs.3.rs-1339051/v1

License: (a) (i) This work is licensed under a Creative Commons Attribution 4.0 International License.

Read Full License 


\section{Abstract}

Background: The immune system is recognized to have therapeutic potential to destroy cancer cells. Tcell immunoglobulin mucin domain-3 (sTIM-3) and its ligand galectin 9 (GAL9) cause suppression of cytokine production, cell cycle arrest, and cell death. Then, soluble levels of TIM-3 and GAL-9 may have prognostic implications in non-small-cell lung cancer (NSCLC) in non-smoker patients.

Methods: This prospective cohort study, including 49 NSCLC patients with an age median of 65 years, evaluated the soluble levels of sTIM-3 and SGAL9 by enzyme-linked immunosorbent assay.

Results: Low levels of sGAL9 in smokers compared to non-smoker NSCLC patients $(P<0.0001)$. Overall survival (OS) analysis showed a low median survival of 10.5 months in smokers NSCLC patients compared to non-smokers (Log-Rank test; $P=0.009$ ). The area under the curve (AUC) value of the Receiver Operating Characteristic (ROC) curve was 0.8497 , suggesting that SGAL9 could be an OS biomarker for advanced NSCLC, being the cut-off value of serum of $1,694 \mathrm{pg} / \mathrm{mL}$ for sGAL9. Low sGAL9 levels NSCLC patients' group had significantly shorter OS than those in the high sGAL9 levels (Log-Rank test; $\mathrm{P}=0.02$ ).

Conclusion: This study indicated the accuracy of SGAL9 as a potential biomarker predictive of OS in advanced NSCLC.

\section{Introduction}

Lung cancer (LC) is the second most frequent type of cancer and the principal cause of death worldwide. Globally, in 2020, around 2.2 million new cases were reported and almost 1.8 million deaths [1]. More than $85 \%$ of patients are classified as non-small-cell lung cancer (NSCLC). Its high incidence is strongly related to smoking, implicated in $80 \%-85 \%$ of cases [2,3]. Cancer immunotherapy by immune-checkpoint has demonstrated a consistent response and increase in overall survival. The therapy with monoclonal antibodies antagonist of the program death-cell 1 (PD1) and its ligand (PDL1) receptors showed wellaccepted in LC patients, but not in all patients [4].

New inhibitory targets of the immune system response have been identified with potential anticancer effects. T-cell immunoglobulin mucin domain-3 (TIM-3) is another immune checkpoint as a T-cell activation/exhaustion marker by interferon- $叉$ producing $T$ cells when expressed on the cell membrane. The galectina-9 (GAL-9) is a ligand of the TIM-3 receptor. It can stimulate it to regulate the immune response, promote $\mathrm{CD} 8+\mathrm{T}$ cell exhaustion, expand myeloid-derived suppressor cells, and improve the immunosuppressive capacity for tumor progression [5]. The potential clinical of TIM-3 and Gal-9 has been identified in patients with lung cancer from tumor tissue or tumor-infiltrating lymphocytes [6-8].

Cigarette smoking seems to compromise the host inflammation response [9] and induce immune dysfunction in the lungs [10]. Smoking increases the number of alveolar macrophages and stimulates the production of pro-inflammatory mediators, reactive oxygen species, and proteolytic enzymes that could 
cause different kinds of tissue damage [9]. Smoking could alter cancer signaling pathways and the tumor microenvironment [10]. When used to identify biomarkers in patients with LC, blood samples could be cost-effective, easily collected, operator-independent, and rapid [11]. The identified biomarker can predict the disease or the evolution process. Higher soluble TIM-3 was associated with poor survival [12].

However, levels of soluble TIM-3 (sTIM-3) and GAL-9 (sGAL-9) in NSCLC need to be evaluated because they can be potential biomarkers and immune-stimulating targets for prognosis or treatment. Thus, in this study, we aimed to assess the expression of STIM-3 and SGAL-9 in the blood of advanced NSCLC patients.

\section{Methods}

\subsection{Study Design}

This cohort study was performed at Instituto de Medicina Integral Prof Fernando Figueira (IMIP), Recife/PE and Universidade de São Paulo (UNIFESP), São Paulo/SP. The institutional review board of IMIP (No 29195220.1.0000.5505) approved the study protocol. Forty-nine patients were diagnosed with advanced NSCLC from January 2019 to January 2020.

Peripheral blood samples were collected during the patients' initial appointments and those without ongoing cancer treatment. Smoking status was categorized as a non-smoker, defined as patients who had never smoked or ex-smokers who had stopped smoking more than 24 months before the admission date. Smokers are patients who still smoke or had stopped smoking in less than six months before the admission date. Tumor histopathology was estimated according to World Health Organization (WHO) and TNM (tumor, node, and metastasis) staging according to UICC (International Union Against Cancer) classifications $[13,14]$.

\subsection{Enzyme-linked immunosorbent assay (ELISA)}

Expression of soluble TIM-3 (sTIM-3) and SGAL-9 were determined by the enzyme-linked immunosorbent assay (ELISA) method, with commercially available antibodies (R\&D Systems, Minneapolis, USA). Absorption was read at $450 \mathrm{~nm}$ using an enzyme immunoassay plate reader (BioTek Instruments) with the wavelength correction set to $540 \mathrm{~nm}$.

\subsection{Statistical Analysis}

Data analysis was performed using GraphPad Prism program v8.0. A descriptive analysis was performed using mean and standard deviation, median and interquartile. The Chi-square test and Fischer's exact test were used to compare the distribution of the categorical variables, and any cases with missing data were excluded from the analysis. A non-parametric test was used to compare two groups (Mann-Whitney $\mathrm{U}$ test) and t student test for mean and standard deviation analysis. Kaplan-Meier survival curves with logrank tests were used to estimate overall survival (OS). The receiver operating characteristic (ROC) curves were up to evaluate the predictive value of sGAL-9 and sTIM-3. The sGAL9 and STIM3 ROC were 
performed in NSCLC patients who died versus survivors. The specific cut-off for GAL9 was calculated by ROC analysis. Differences were considered statistically significant when $P$ values were $<0.05$.

\section{Results}

\subsection{Clinical variables}

This analysis included 49 patients with advanced NSCLC with an age median of 65 years and were 26 men (53.1\%) and 23 women (46.9\%). Twenty-three smokers (47\%) and 26 non-smokers (53\%). The latter had a more significant smoking load: $64.2 \%$ smoked more than 30 packs/year. The adenocarcinoma type was the most frequently diagnosed (45.0\%), followed by undifferentiated $(28.5 \%)$ and squamous cell carcinoma (26.5\%). Forty-two patients (85.7\%) were stage IV, and $14.3 \%$ were stage III (Table 1 ).

Table 1. Characteristics of the 49 patients with non-small cell lung cancer (NSCLC)

\begin{tabular}{|lll|}
\hline Variables & N=49 & \\
\hline Age & & \\
\hline Median (IQR). & $65.0(54.0-75.0)$ & \\
\hline & N & \\
\hline SEX & & 53.1 \\
\hline Male & 26 & 46.9 \\
\hline Female & 23 & \\
\hline HISTOLOGY TYPE & & 45.0 \\
\hline Adenocarcinoma & 22 & 26.5 \\
\hline Squamous Cell Carcinoma & 13 & 28.5 \\
\hline Undifferentiated & 14 & \\
\hline TNM & & 14.3 \\
\hline III & 7 & 85.7 \\
\hline IV & 42 & 47.0 \\
\hline SMOKERS & & 53.0 \\
\hline Yes & 23 & \\
\hline No & 26 & \\
\hline
\end{tabular}

TNM: tumor-node-metastasis system; IQR: interquartile 


\section{2 sGAL9 and sTIM-3 levels in NSCLC patients}

Low levels of sGAL9 in smokers compared to non-smoker NSCLC patients $(p<0.0001)$. There were no significant differences in SGAL-9 levels between age $\leq 65>65$ years, sex, and histopathology types

(Figure 1). There were no significant differences in STIM-3 levels between smokers and non-smoker, age $\leq 65>65$ years, sex, and histopathology types (Figure 2).

\subsection{Overall survival in NSCLC patients}

Overall survival (OS) analysis showed a low median survival of 10.5 months in smokers NSCLC patients compared to non-smokers (Log-Rank test; $P=0.009$; Figure 3).

By using the ROC curve, we found that a baseline of $1694 \mathrm{pg} / \mathrm{ml}$ (cutoff). sGAL9 with specificity (72.2\%), sensitivity $(83.2 \%)$, and area under the curve - AUC $=0.8497(P<0.0004)$ for differentiating the median overall survival in advanced NSCLC (Figure 4). Low sGAL9 levels NSCLC patients' the group had significantly shorter OS, a median survival of 15 months, than those in the high sGAL9 levels (Log-Rank test; $P=0.02$ ). AUC sTIM3 were of 0.64 , were not statistically significant in the advanced NSCLC (Figure $5)$.

\section{Discussion}

Thus, this study showed that changes in SGAL-9 levels were associated with smoking and shorter overall survival in NSCLC. Galectins contribute to carcinogenesis and cancer progression, including cancer, proliferation, metastasis, angiogenesis, and the immune response [15].

This study also showed high sGAL9 and better OS in non-smokers NSCLC patients than smokers. Tobacco smoke contains many chemical and carcinogenic particles that could develop chronic pulmonary inflammation and lung cancer. Curiously, the lung cancer of smokers and non-smokers have distinct inflammatory signatures, with marked differences in mast cell and CD4+ T cell numbers. Furthermore, high regulatory T cells, even at early stages, and reduced CD8+ T cells in the tumor lesion; have been related as an immune escape mechanism in NSCLC patients [10].

High sGAL9 levels were associated with a better prognosis in OS analysis compared to the patients' group with low sGAL9 levels. Thus, we can infer that smoking not only could damage immune system processes due to chronic pulmonary inflammation, with reduction of SGAL9 levels but also favors the onset of LC and its worse prognosis. A limited number of studies have investigated the role of GAL9 in lung cancer. Increased GAL9 expression in tumor cells may suppress pulmonary metastasis, recurrence of melanoma, and breast cancer [16].

Kadowaki et al. 2013 shown in lung carcinoma cell-bearing mice that GAL9 expression induces macrophage differentiation into plasmacytoid dendritic cell-like macrophages, which may augment the 
activation of NK cells and was associated with, increased the survival of tumor-bearing mice [17]. Only $15 \%-20 \%$ of NSCLC patients under immune checkpoint inhibitor treatment achieve a partial or complete response. The resistance mechanism to immunotherapy needs to be better-understood [18]. One study showed that early accumulation of monocytic myeloid-derived suppressor cells expressing GAL9 [18].

The analysis of expression of GAL-9 is on NSCLC tumor cells, and tumor-infiltrating lymphocytes (TILs) showed that low GAL9 levels on tumor cells or high GAL-9 levels on TILs were more likely to have a poor prognosis. GAL-9 on TILs correlated with TIM-3, PD-1, and PD-L1 levels, and on tumor cells, GAL-9 was associated with the expression of TIM-3 [19]. D'Alessandro et al. 2020 demonstrated that Galectins 1, 3, and 9 levels have been changed in serum idiopathic pulmonary fibrosis, suggesting their potential utility as clinical, diagnostic, and prognostic biomarkers [20].

In NSCLC patients, high TIM-3 and GAL-9 levels correlated with larger tumor sizes, advanced stages, and more distant metastasis [21]. Lymphoid cells with high TIM-3 expression are associated with primary and secondary resistance in metastatic LC patients under anti-PD-1 treatment [21]. Moreover, TIM-3 is often co-expression with PD1 in exhausted CD8 cells in infections and tumors, which could explain why an isolated blockade of TIM-3 could be effective [21].

This study indicated the predictive accuracy of SGAL9 in the survival advanced NSCLC. We suggest that changes in SGAL 9 levels could be associated with smoking and deaths in lung cancer. However, an increase of GAL9 levels in the serum of non-smokers patients was associated with a better OS in advanced NSCLC. In conclusion, SGAL9 is a potential biomarker predictive of OS in advanced NSCLC.

\section{Declarations}

\section{Author Contributions Statement}

Author contributions: All authors have participated directly and wrote the manuscript, approved the version to be published, and agreed to be accountable for all aspects of the work. Guilherme Jorge Costa: Conceptualization; investigation, methodology, data curation, formal analysis, funding acquisition, data interpretation, and writing - original draft. Guilherme Vieira de Mendonça Filho: Investigation, writing original draft, and data curation; Leuridan Cavalcante Torres: Investigation, methodology, formal analysis, funding acquisition, visualization, resources, and writing - review \& editing; Dulce Elena Casarini: Supervision, project administration, investigation, data curation, and writing - review \& editing.

\section{ACKNOWLEDGEMENT}

We want to thank all the patients. This project would not have been possible without the organization and help from Prof. Jurema Telles de Oliveira Lima, Prof. Carla Rameri, Alexandre Silva de Azevedo, and Mrs. Gerlane Albuquerque dos Santos e Silva. 
FINANCIAL SUPPORT: This study was supported by grants from Ministério da Saúde, Programa Nacional de Apoio à Atenção Oncológica (PRONON, Grants Number: 25000.158.891/2014-27), Conselho Nacional de Desenvolvimento Científico e Tecnológico, Brazil (CNPq; Grants Number: 420739/2018-1) and Fundação de Amparo à Pesquisa do Estado de São Paulo (FAPESP; grant number 2017/17027-0)

CONFLICT OF INTEREST: None of the authors has any potential financial conflict of interest related to this manuscript.

\section{References}

1. Sung H, Ferlay J, Siegel RL, et al. Global Cancer Statistics 2020: GLOBOCAN Estimates of Incidence and Mortality Worldwide for 36 Cancers in 185 Countries. CA Cancer J Clin. 2021;0:1-41.

2. Alberg AJ, Brock MV., Ford JG, Samet JM, Spivack SD. Epidemiology of lung cancer: Diagnosis and management of lung cancer, 3rd ed: American College of Chest Physicians evidence-based clinical practice guidelines. Chest. 2013;143:1-29.

3. Costa GJ, Júlia M, Mello G De, et al. Lung cancer increased incidence, morbidity and mortality rates for lung cancer in women in Brazil between 2000 and 2014 : An analysis of three types of sources of secondary data. Lung Cancer. 2018;125(March):77-85.

4. Ettinger DS, Wood DE, Aisner DL, Akerley W, Bauman JR, Bharat A, Bruno DS, Chang JY, Chirieac LR, D'Amico TA, Dilling TJ. Non-small cell lung cancer, Version 2.2021 featured updates to the NCCN guidelines. JNCCN Journal of the National Comprehensive Cancer Network. 2021;19(3):254-66.

5. Du W, Yang M, Turner A, et al. TIM-3 as a target for cancer immunotherapy and mechanisms of action. Int J Mol Sci. 2017;18(645):1-12.

6. Jia K, He Y, Dziadziuszko R, et al. T cell immunoglobulin and mucin-domain containing-3 in nonsmall cell lung cancer. Transl Lung Cancer Res. 2019;1(6):895-906.

7. He Y, Jia K, Dziadziuszko R, et al. Lung Cancer Galectin-9 in non-small cell lung cancer. Lung Cancer. 2019;136:80-85.

8. Dempke WCM, Fenchel K, Uciechowski P, Dale SP. Second- and third-generation drugs for immunooncology treatment-The more the better ? Eur J Cancer. 2017;74:55-72.

9. Benevides L, Gonc RB, Coletta RD, Silve KG, Casati MZ. Impact of smoking on inflammation: overview of molecular mechanisms. Inflamm Res. 2011;60:409-424.

10. Li X, Li J, Wu P, Zhou L, Lu B, Ying K, Chen E, Lu Y, Liu P. Smoker and non-smoker lung adenocarcinoma is characterized by distinct tumor immune microenvironments. Oncoimmunology. 2018;7(10):1-11.

11. Mamdani H, Ahmed S, Armstrong S, Mok T, Jalal SI. Blood-based tumor biomarkers in lung cancer for detection and treatment. Transl Lung Cancer Res. 2017;6:648-660.

12. Li F, Li N, Sang J, Fan X, Deng H, Zhang X, Han Q, Lv Y, Liu Z. Highly elevated soluble Tim-3 levels correlate with increased hepatocellular carcinoma risk and poor survival of hepatocellular carcinoma patients in chronic hepatitis B virus infection. Cancer Manag Res. 2018;10:941-951. 
13. Goldstraw P, Chansky K, Crowley J, et al. The IASLC lung cancer staging project: Proposals for revision of the TNM stage groupings in the forthcoming (eighth) edition of the TNM Classification for lung cancer. J Thorac Oncol. 2016;11:39-51.

14. Travis WD, Brambilla E, Nicholson AG, et al. The 2015 World Health Organization classification of lung tumors: Impact of genetic, clinical and radiologic advances since the 2004 classification. $J$ Thorac Oncol. 2015;10:1243-1260

15. Pinho SS, Reis CA. Glycosylation in cancer: Mechanisms and clinical implications. Nat Rev Cancer. 2015;15:540-555.

16. Nobumoto A, Nagahara K, Oomizu S, Katoh S, Nishi N, Takeshita K, Niki T, Tominaga A, Yamauchi A, Hirashima M. Galectin-9 suppresses tumor metastasis by blocking adhesion to endothelium and extracellular matrices. Glycobiology. 2008;18:735-744.

17. Kadowaki T, Arikawa T, Shinonaga R, Oomizu S, Inagawa H, Soma G, Niki T, Hirashima M. Galectin-9 signaling prolongs survival in murine lung-cancer by inducing macrophages to differentiate into plasmacytoid dendritic cell-like macrophages. Clin Immunol. 2012;142:296-307.

18. Mazzarella L, Achutti B, Trapani D, et al. The evolving landscape of 'next-generation' immune checkpoint inhibitors: A review. Eur J Cancer. 2019;117:14-31.

19. He Y, Jia K, Dziadziuszko R, Zhao S, Zhang X, Deng J, Wang H, Hirsch FR, Zhou C. Galectin-9 in nonsmall cell lung cancer. Lung Cancer. 2019;136:80-85.

20. D'Alessandro M, De Vita E, Bergantini L, Mazzei MA, di Valvasone S, Bonizzoli M, Peris A, Sestini P, Bargagli E, Bennett D. Galactin-1, 3 and 9: Potential biomarkers in idiopathic pulmonary fibrosis and other interstitial lung diseases. Respir Physiol Neurobiol. 2020;282:103546.

21. Limagne E, Richard C, Thibaudin M, Fumet J, Truntzer C. Tim-3 / galectin-9 pathway and mMDSC control primary and secondary resistance to PD-1 blockade in lung cancer patients. Oncoimmunology. 2019;8:1-13.

\section{Figures}




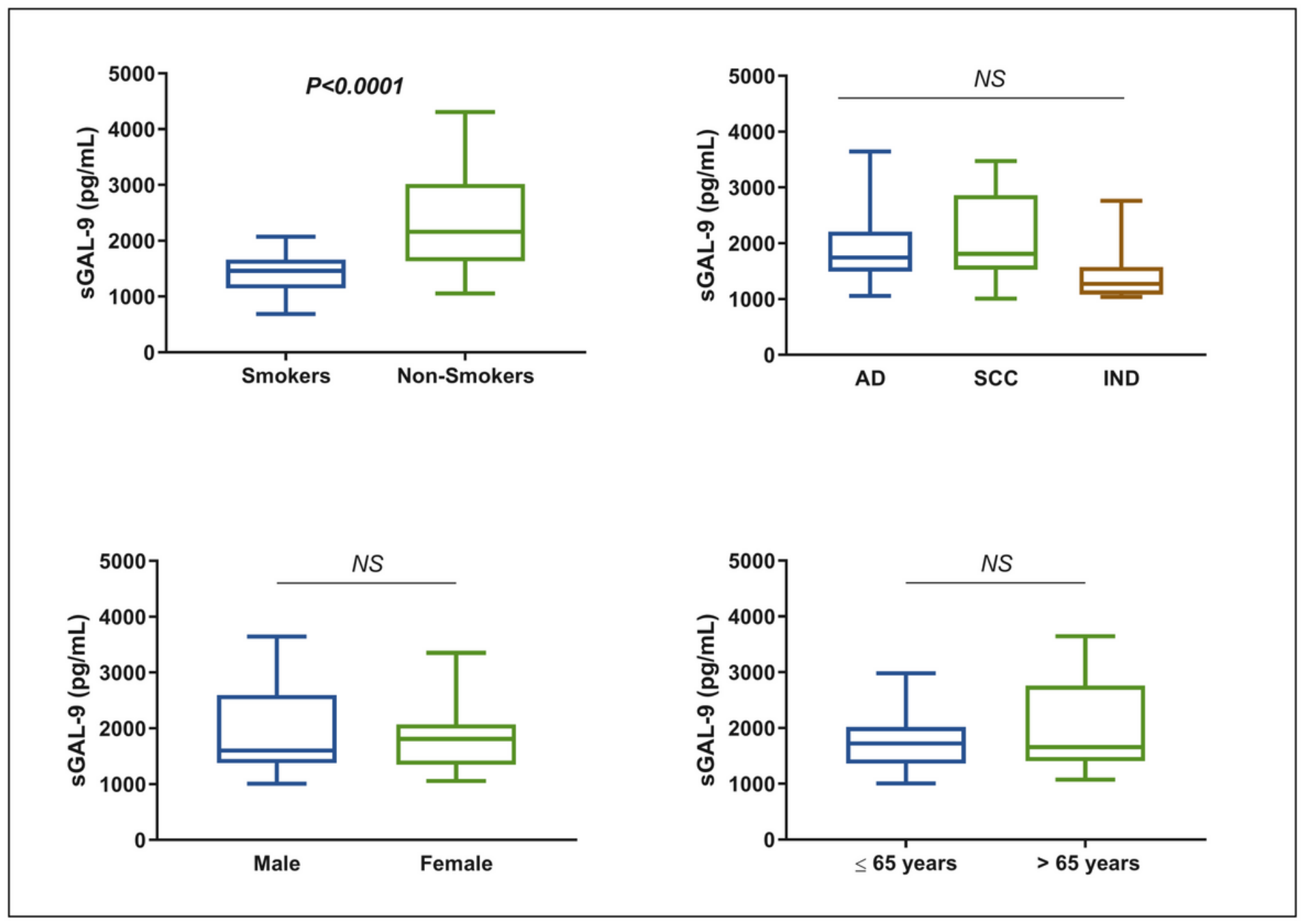

Figure 1

sGAL-9 levels in advanced NSCLC patients, accordingly, smoking status, age, sex, and histopathology types. The graphs are represented in median and interquartile. The Mann Whitney test was performed. It was considered significant $p<0.05$. NS: Not significant. Adenocarcinoma (ADD), Squamous cell Carcinoma (SCC) and Undifferentiated (UND). 

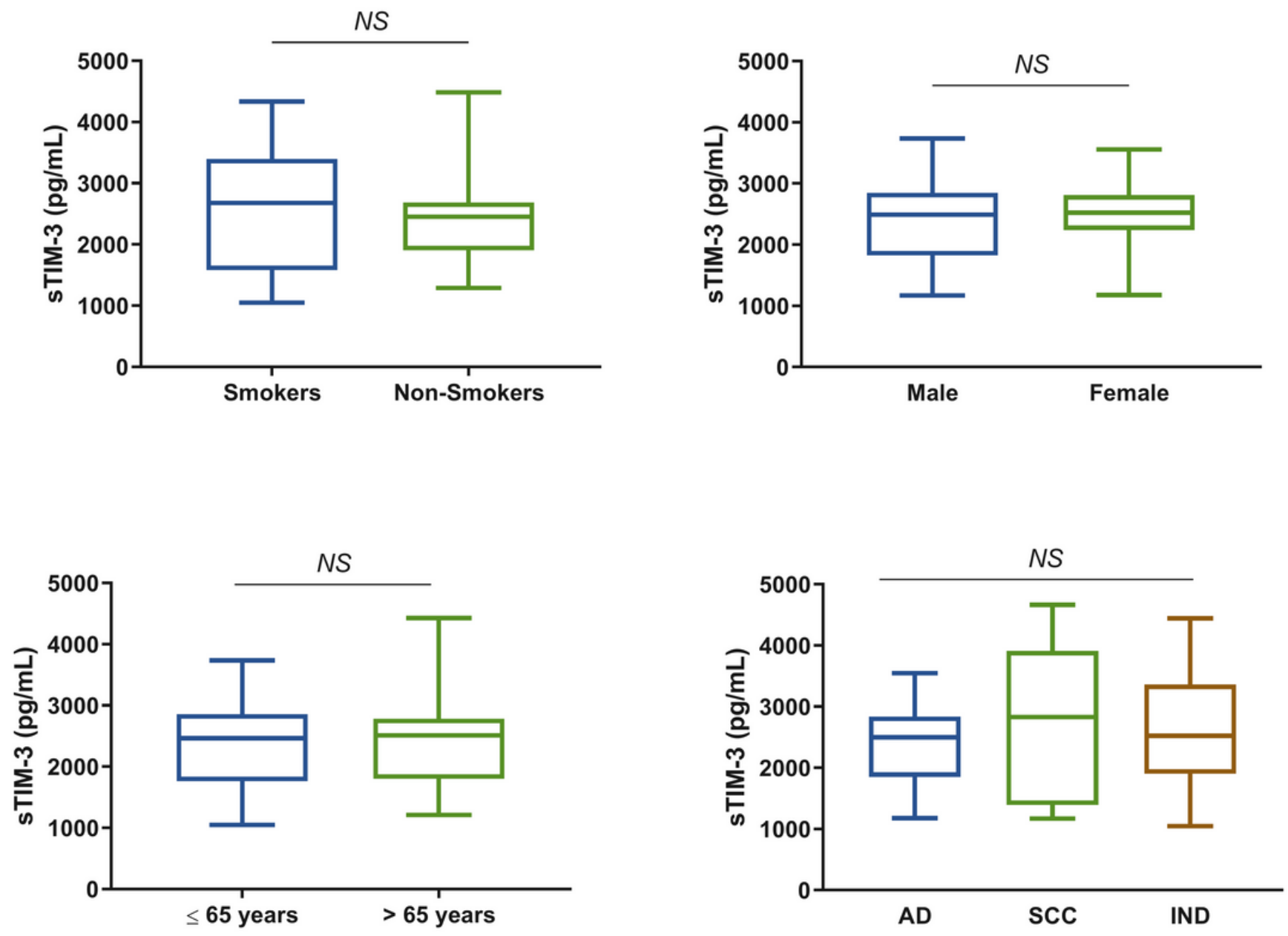

Figure 2

sTIM3 levels in advanced NSCLC patients, accordingly, smoking status, age, sex, and histopathology types. The graphs are represented in median and interquartile. The Mann Whitney test was performed. It was considered significant $p<0.05$. NS: Not significant. Adenocarcinoma (ADD), Squamous cell Carcinoma (SCC) and Undifferentiated (UND). 

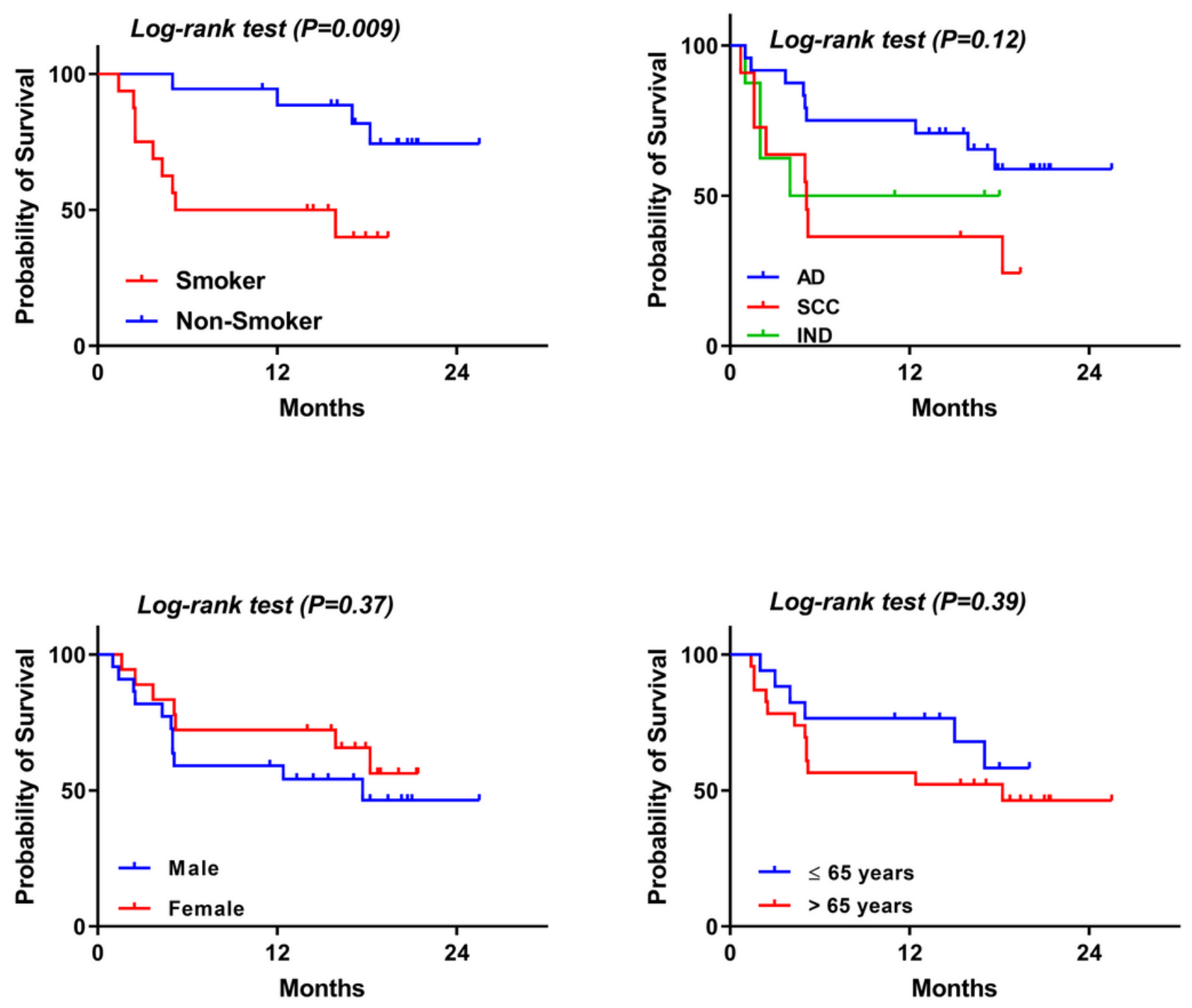

Figure 3

The association between smoking status, age, sex, and histopathology types with overall survival of NSCLC patients. Adenocarcinoma (ADD), Squamous cell Carcinoma (SCC) and Undifferentiated (UND). 
ROC curve: ROC of GAL9

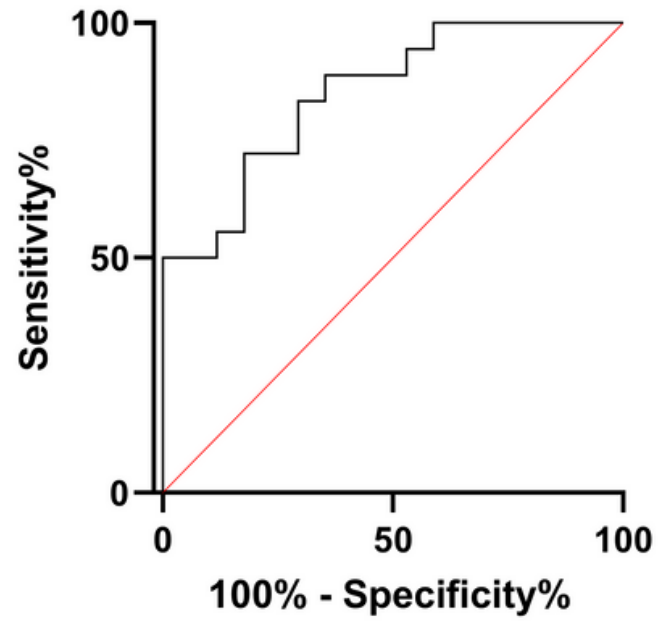

Area

Std. Error

95\% confidence interval

$P$ value

\begin{tabular}{l}
0.8497 \\
0.06310 \\
0.7260 to 0.9733 \\
0.0004 \\
\hline
\end{tabular}

ROC curve: ROC of TIM3

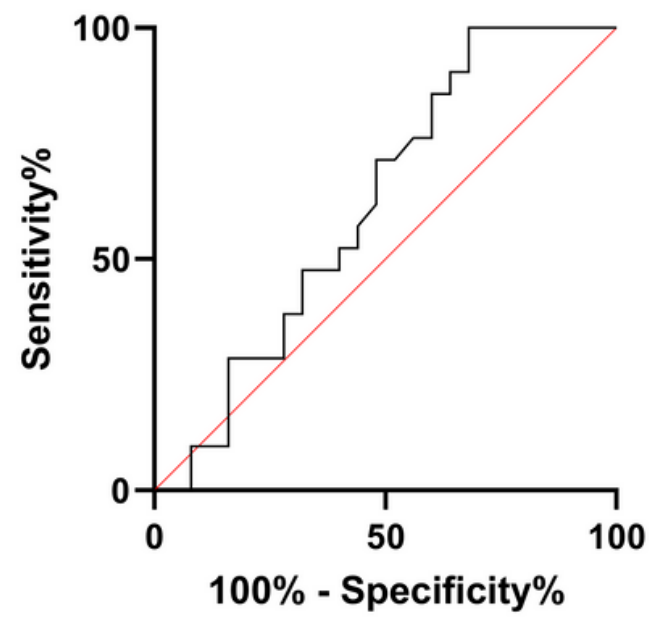

\begin{tabular}{|l|l|}
\hline Area & 0.6190 \\
Std. Error & 0.08316 \\
\hline $95 \%$ confidence interval & 0.4561 to 0.7820 \\
P value & 0.1681 \\
\hline
\end{tabular}

Figure 4

ROC analysis evaluating prognosis accuracy of serum SGAL9 and STIM3 in advanced NSCLC. 


\section{Log-rank test $(P=0.02)$}

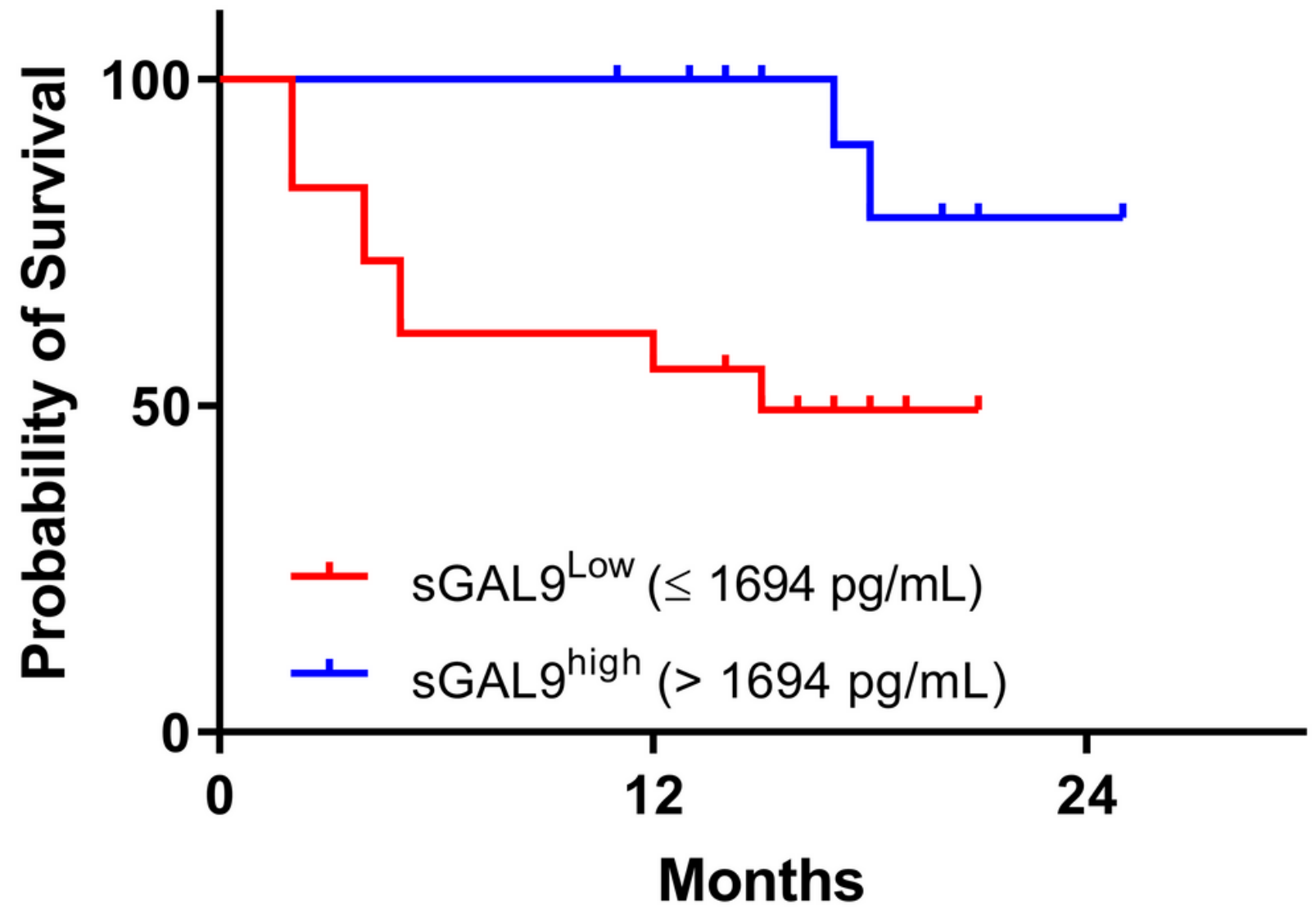

Figure 5

Prognostic effects of sGAL9 levels on overall survival of advanced NSCLC patients. 